\title{
Aggression as a function of exposure and similarity to a nonaggressive model $^{*}$
}

\author{
DAVID M. WALDMAN and ROBERT A. BARON† \\ University of South Carolina, Columbia, S.C. 29208
}

Eighty male undergraduates participated in an experiment designed to investigate the effects of exposure to a nonaggressive model, level of prior anger arousal, and degree of observer-model similarity on adult aggressive behavior. The dependent measures of aggression were the intensity and duration of shocks delivered to the victim by Ss on occasions when this person appeared to make errors on a learning task. Results indicated that exposure to the model was effective in reducing the duration of attacks against the victim both in the presence and absence of prior anger arousal, but succeeded in lowering the intensity of Ss' aggressive acts only in the absence of such instigation.

Informal observation suggests that in many tense and potentially dangerous situations, the occurrence of overt violence is frequently averted by the presence of individuals who both demonstrate and verbally urge restraint. Within the context of social learning theory (e.g., Bandura, 1965), such persons may be viewed as nonaggressive models who, by their actions and verbalizations, strengthen the inhibitions of potential aggressors against engaging in overt aggressive acts.

Although it is apparent that such individuals may play an important role in the prevention and control of human violence, relatively little attention has been directed to their potentially beneficial influence. Indeed, only a few experiments have been directly concerned with this issue (e.g., Baron \& Kepner, 1970; Walters \& Willows, 1968), and even these studies have provided relatively inconclusive results. Thus, the primary purpose of the present experiment was that of attaining additional information on the possible aggression-inhibiting influence of live nonaggressive models. More specifically, attention was focused on the question of whether exposure to such models would be effective in inhibiting subsequent violence by adult observers, even under conditions where they had previously been subjected to strong provocation from the target of their attacks.

It has often been suggested (e.g., Bandura \& Walters, 1963) that imitative behavior is facilitated by a high degree of similarity between observers and the model. The results of several recent studies (e.g., Rosekrans, 1967) lend direct support

*The authors wish to express their appreciation to John MacDonald and John Mullins for their able assistance in the collection of the data.

tRequests for reprints should be sent to Robert A. Baron, who is now at the Department of Psychology, Purdue University, Lafayette, Indiana $\mathbf{4 7 9 0 7}$ to this suggestion. Thus, a second purpose of the present experiment involved the investigation of degree of observer-model similarity as a factor which might affect the magnitude of any restraining influence of nonaggressive models. In accordance with the findings of previous research concerned with this factor, it was tentatively suggested that exposure to a nonaggressive model would be more effective in inhibiting subsequent violence by observers under conditions of high than under conditions of low apparent similarity to the model.

\section{SUBJECTS}

Eighty undergraduate males enrolled in summer session courses at the University of South Carolina participated in the experiment. Forty of these individuals took part in the study to earn extra course credit, while the remaining individuals participated in order to earn a $\$ 2$ payment.

\section{DESIGN AND APPARATUS}

A 2 by 2 by 2 factorial design based upon two levels of similarity to the model (low, high), two levels of prior anger arousal (nonangry, angry), and two levels of model's behavior (no model, nonaggressive model) was employed. Ten Ss, half of whom participated in the study for course credit and half for the monetary payment, were assigned randomly to each cell of this design.

The apparatus consisted of a modified Buss "aggression machine" (Buss, 1961), a Lafayette stop clock (Model 5721), an intercom system, a Hunter interval timer (Model 111-C), and a device for the delivery of shock to Ss.

\section{PROCEDURE}

When Ss arrived for the study, they found two confederates of the $\mathrm{Es}(\mathrm{Cl}$ the future victim, and $\mathrm{C2}$, the future model) already present. Shortly thereafter, the $E$ arrived and conducted all three individuals to the first experimental room, where systematic manipulations of degree of observer-model similarity and level of prior anger arousal experienced by Ss were attempted. Brief descriptions of the procedures employed for these purposes follow.

\section{Apparent Similarity \\ to the Model}

Apparent similarity between the $S$ and model was manipulated by varying the extent to which these individuals appeared to share a common social background. Thus, in the low-similarity condition, the model's dress (a gray work uniform) and answers to a short questionnaire suggested that he came from a background very different from $S$ 's, while in the high-similarity condition, the model's dress (typical student attire) and answers to the questionnaire indicated that he came from a background very similar to that of the $S^{1}$

Following the completion of the above procedures, the level of anger arousal experienced by $S$ s was manipulated. The procedures employed for this purpose were highly similar to those employed in previous research (e.g., Baron, 1971) and will not be described in detail here. Briefly, the $\mathrm{S}$ and model (i.e., $\mathrm{C} 2$ ) were asked to offer written solutions to a pressing social problem (how to control rising crime rates), and were then presented with evaluations of their work from C1 (the future victim). In the nonangry condition, these evaluations were very favorable and complimentary, while in the angry condition they were quite insulting and unfavorable. In both conditions, Ss were uninformed as to the type of evaluation received by the model.

Aggression Opportunity and Exposure to the Model

After the above procedures were concluded, the $E$ explained that all three individuals would now participate in a final phase of the experiment designed to investigate the effects of punishment on learning. During this portion of the study, the $S$ and model were assigned the role of teachers and instructed to punish $\mathrm{C} 1$, who played the role of learner, with electric shocks on each occasion when he made an error in a paired-associates learning task. The $\mathrm{E}$ explained that the teachers could control both the intensity and duration of these shocks by means of a series of 10 "shock buttons" on the experimental apparatus, and that they were free to use any level of punishment they wished. Both the $\mathrm{S}$ and the model were then administered sample shocks from Buttons 4 and 5 (36 and $50 \mathrm{~V}$ respectively) to convince them that the "aggression machine" was, in fact, operational. After administering the 


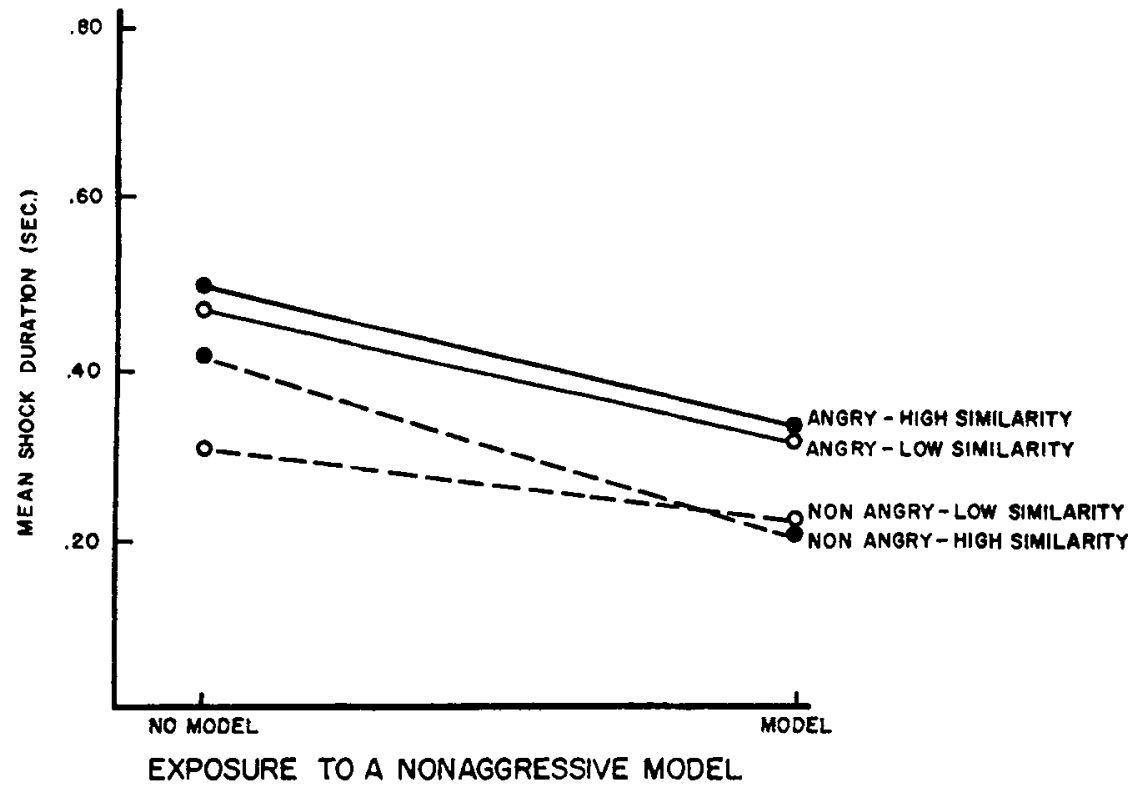

Fig. 1. Mean duration of shocks delivered to the learner by Ss in each of eight experimental groups.

sample shocks, the $\mathrm{E}$ selected either the $\mathbf{S}$ or the model to serve as the first teacher. In the nonaggressive-model condition, C2 was chosen to play this role, while in the no-model condition, the $S$ was designated as the first teacher. Under both conditions, the model acted in a nonaggressive manner when it was his turn to serve as teacher, punishing errors by the learner with shocks from Buttons 1, 2, or 3 on the experimental apparatus. Since the model responded before the $S$ only in the nonaggressive-model

condition, however, it is apparent that he could influence the $S$ 's behavior only in this condition. The learner (i.e., C1) appeared to make a total of 20 errors during the session. RESULTS

Shock Duration

Figure 1 presents the mean duration of shocks administered to the learner by $\mathrm{Ss}$ in each of the eight experimental groups. ${ }^{2}$ Inspection of this figure suggests that Ss exposed to the model before aggressing delivered shorter shocks to the learner than Ss not

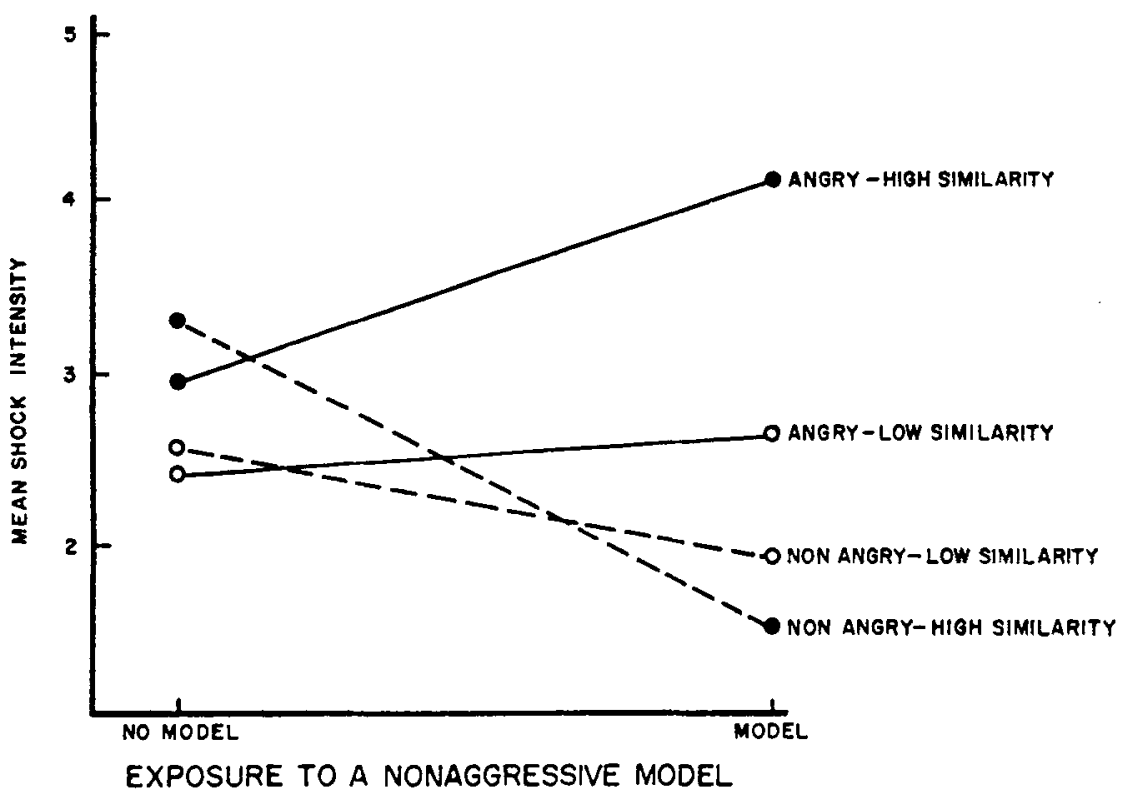

Fig. 2. Mean intensity of shocks delivered to the learner by Ss in each of eight experimental groups. exposed to the model, and that Ss in the angry condition delivered longer shocks to this individual than did Ss in the nonangry condition.

An analysis of variance performed on these data indicated that the main effects of exposure to the model $(F=14.10, \quad \mathrm{df}=72, \quad \mathrm{p}<.001)$ and anger arousal $(F=9.24, \quad \mathrm{df}=1 / 72$, $p<.005$ ) were both significant. Thus, as suggested by Fig. 1, aggression was facilitated by anger arousal, but inhibited by exposure to the nonaggressive model.

\section{Shock Intensity}

Figure 2 presents the mean intensity of shocks administered to the learner by Ss in each of the eight experimental groups. Inspection of this figure suggests that in contrast to the findings for the duration measure, exposure to the model was successful in reducing aggressive behavior only under conditions where Ss had not been angered by the victim of their attacks.

An analysis of variance performed on these data indicated that, as suggested by Fig. 2, the interaction between anger arousal and exposure to the model was significant $(F=6.83$, $\mathrm{df}=1 / 72, \mathrm{p}<.025$ ). Follow-up simple effects analyses, performed to examine this interaction more closely, revealed that exposure to the model significantly reduced the level of aggression shown by $S s$ in the nonangry groups $(F=5.39$, $\mathrm{df}=1 / 72$, $p<.025$ ), but failed to exert a significant effect upon such behavior in the angry condition $(F=1.90$, $\mathrm{df}=1 / 72, \mathrm{p}>.05)$. Thus, aggression was inhibited by exposure to a nonaggressive model only under conditions where $S s$ had not previously been exposed to strong provocation from the victim. DISCUSSION

The two dependent measures of aggression employed in the present study yielded somewhat different patterns of results. Specifically, findings for the duration measure suggested that exposure to a nonaggressive model may be highly effective in reducing subsequent aggression by observers both in the presence and absence of prior anger arousal, while findings for the intensity measure suggested that such effects may occur only in the absence of provocation from the victim. One possible explanation for these contrasting patterns of results may be derived if it is assumed that these two dependent measures are primarily sensitive to different aspects or components of aggression. In particular, it may be tentatively suggested that shock intensity reflects primarily Ss' conscious intent to harm the victim, while shock duration is 
more directly sensitive to the emotional arousal which often accompanies overt acts of violence (see, e.g., Baron \& Kepner, 1970). Thus, in the present study, exposure to the calm, nonemotional model may have served to lower Ss' own emotional arousal, but failed to reduce their desire to inflict pain and suffering on the victim. As a result, the model was effective in reducing the duration but not the intensity of attacks against the anger instigator. Direct evidence concerning these suggestions may be obtained in future research by relating verbal reports concerning Ss' desire to harm the victim to the intensity of the shocks they employ, and relating physiological measures of emotional arousal to the duration of such attacks.

In contrast to initial predictions, degree of observer-model similarity failed to affect the magnitude of the aggression-inhibiting influence of the model. Thus, it appears that degree of apparent similarity in social background to a nonaggressive model is not an important determinant of the adoption of similar patterns of restrained behavior by adult observers. However, future research is needed to determine whether similarity along any of several additional dimensions (e.g., attitudes, age, sex, race, personality) may be of greater importance in this regard.

\section{REFERENCES}

BANDURA, A. Vicarious processes: A case of no-trial learning. In $L$. Berkowitz (Ed.). Advances in experimental social psychology. New York: Academic Press, 1965

BANDURA, A., \& WALTERS, R. H. Socia learning and personality development. New York: Holt, Rinehart, \& Winston, 1963.

BARON, R. A. Magnitude of victim's pain cues and level of prior anger axousal as determinants of adult aggressive behavior. Journal of Personality \& Social Psychology, 1971, 17, 236-243.

BARON, R. A., \& KEPNER, C. R. Model's behavior and attraction toward the model as determinants of adult aggressive behavior. Journal of Personality \& Social Psychology, 1970, 14, 335-344.

BUSS, A. H. The psychology of aggression. New York: Wiley, 1961 .

ROSEKRANS, M. A. Imitation in children as a function of perceived similarity to a social model and vicarious reinforcement Journal of Personality \& Social Psychology, 1967, 7, 307-315.

WALTERS, R. H., \& WILLOWS, D. C Imitative behavior of disturbed and non-disturbed children following exposure to aggressive and nonaggressive models. Child Development, 1968,39 , 79-88.

\section{NOTES}

1. In both groups, the answers of $\mathrm{Cl}$ suggested that he was moderately similar in social background to the $S$.

2. A preliminary analysis indicated that there were no differences between the paid and unpaid Ss. Thus, their data were combined in all subsequent analyses for both dependent measures.
Solution of the transverse

\section{patterning problem: Response to cue-cue relations}

\author{
DANIEL B. BERCH and MICHAEL ISRAEL \\ California State College, Los Angeles, Calif. 90032
}

The transverse patterning problem was studied in adult human Ss. As hypothesized, the addition of a nonspatial dimension varying among the settings comprising the cue-cue patterns decreased the difficulty of this discrimination task. Contrary to Spence (1952), a number of Ss solved the problem by means of responding to the cue-cue patterns, even though the additional nonspatial dimension permitted solution on the basis of a less complex type of pattern.

Spence (1952) devised a problem which cannot be solved on the basis of responding either to a single reinforced component, as in the simultaneous discrimination, or to a cue-position compound, as in the standard successive problem. This "transverse patterning" discrimination, as Spence (1952) referred to it, consists of three different stimuli presented in pairs. According to Spence, solution of this problem requires that the organism approach or not approach a particular stimulus, depending upon what other stimulus is paired with it. In other words, the effective stimulus is a cue-cue relation, or pattern.

As illustrated in Fig. 1. the transverse patterning problem consists of three such cue-cue relations. Since position is an irrelevant dimension in this task, the combined settings, 1 and 4,2 and 5 , and 3 and 6 , comprise the three patterns. Note that any two of these cue-cue patterns differ with respest to only one value on the form dimension. Consequently, anything which would increase the distinctiveness of these patterns should facilitate learning the discrimination. There is evidence that reducing the similarity of the settings comprising the standard successive discrimination facilitates its solution (Lubker, 1969). This has been achieved by increasing the number of nonspatial dimensions varying between the settings of the problem. Similarly, in the oddity discrimination task, increasing the number of nonspatial dimensions along which the reinforced stimulus is odd, decreases the difficulty of this problem (Croll, 1967, Experiment II).

The purpose of the present experiment was to determine if the addition of the nonspatial dimension, color, varying among the settings comprising the three different cue-cue relations, would facilitate solution of the transverse patterning problem.

\section{PROBLEMS}

The transverse patterning problem, as illustrated in Fig. 1, consisted of three black geometric forms presented in pairs. The addition of the color dimension resulted in what will be referred to as the "color pattern" task. This problem consisted of the same pairs of geometric forms, but the settings comprising each of the three cue-cue patterns differed in color. The three colors, red, yellow, and blue, are represented in Fig. 1 by white, horizontal lines, and vertical lines, respectively. The plus signs stand for reinforced and the minus signs for nonreinforced.

\section{MATERIALS}

The stimuli were constructed out of colored posterboard. They included all possible combinations of three forms -triangle, circle, and square-and four colors-black, red, yellow, and blue. The dimensions of the forms were: square, 3.5-in. side; circle, 3.75-in. diam; triangle, 4-in. base, 3.5 -in. height. Two complete sets of stimuli were constructed.

$$
\text { SUBJECTS }
$$

The Ss were 24 students enrolled in an introductory psychology course at California State College, Los Angeles. Twelve Ss (six males and six females) were assigned randomly to each of two groups, one which learned the transverse patterning problem (Group TP) and the other, the color pattern problem (Group CP).

\section{PROCEDURE}

Upon entering the experimental room, the $\mathbf{S}$ was seated across a table from the $E$ and given the following instructions: "I am going to show you some geometric forms, two at a time. Each time, point to the one you think is right, and I will say either 'right' or 